\title{
Synthesis of S-Glycosyl Primary Sulfonamides
}

Marie Lopez, Nicolas Drillaud, Laurent F. Bornaghi and Sally-Ann Poulsen*

Eskitis Institute, Griffith University, Brisbane, Queensland 4111, Australia.

\author{
s.poulsen@griffith.edu.au
}

RECEIVED DATE (to be automatically inserted after your manuscript is accepted if required according to the journal that you are submitting your paper to)

Corresponding Author: S.-A.P. Tel +61 73735 7825, s.poulsen@griffith.edu.au

\begin{abstract}
The synthesis of $S$-glycosyl sulfonamides wherein the primary sulfonamide functional group $\left(-\mathrm{SO}_{2} \mathrm{NH}_{2}\right)$ is directly attached to the anomeric position of a carbohydrate moiety is reported. Our general approach, consists of first introducing a thioacetate group at the anomeric centre of the per-O-acetylated sugar derivative. From this follows formation of a glycosyl sulfenamide (sugar-SNR 2 ), oxidation of the sulfenamide to give a glycosyl $\mathrm{N}$-protected sulfonamide (sugar- $\mathrm{SO}_{2} \mathrm{NR}_{2}$ ), and removal of the sulfonamide protecting $(\mathrm{R})$ group to yield a primary sulfonamide at the anomeric centre (sugar-
\end{abstract}


$\mathrm{SO}_{2} \mathrm{NH}_{2}$ ). A variety of mono- and disaccharide derivatives were synthesized using this new methodology.

\section{Keywords}

primary sulfonamide, carbohydrate, glycosyl sulfonamide, anomeric

\section{Introduction}

The sulfonamide group has proven of remarkable utility in medicinal chemistry and features in the structure of a number of clinically relevant small molecules. ${ }^{1}$ Almost all therapeutically used sulfonamides are aromatic $\left(\mathrm{Ar}-\mathrm{SO}_{2} \mathrm{NH}_{2}\right.$ or $\left.\mathrm{Ar}-\mathrm{SO}_{2} \mathrm{NHR}\right){ }^{2}$ To our knowledge there are no reports of the synthesis of S-glycosyl sulfonamides wherein the primary sulfonamide functional group is linked directly to the anomeric position of a carbohydrate.

The synthesis of primary sulfonamides is typically achieved by the reaction of a substituted sulfonyl chloride with excess ammonia. Anomeric sulfonyl chlorides in principle could serve as precursors to anomeric sulfonamides. Recently Knapp and co-workers ${ }^{3}$ attempted the synthesis of anomeric sulfonyl chlorides with this task in mind. All conditions evaluated in this study resulted instead in the formation of anomeric chlorides as product rather than the desired anomeric sulfonyl chloride. It was proposed that although the anomeric sulfonyl chloride was likely to have formed it proved unstable towards loss of $\mathrm{SO}_{2}$, rapidly converting into the chloride. The lack of stability of precursor anomeric sulfonyl chlorides eliminates this synthetic methodology as an avenue to anomeric sulfonamides. We now present a relatively simple procedure for the preparation of $S$-glycosyl primary sulfonamides. As new chemical entities, we expect anomeric sulfonamides will prove valuable species to investigate biological interactions and other parameters relevant to drug discovery.

\section{Results and Discussion}


Glycosyl sulfenamides (sugar-SNR 2 ) have been prepared and oxidized to glycosyl $N, N$-disubstituted sulfonamides (sugar- $\mathrm{SO}_{2} \mathrm{NR}_{2}$ ) by others. ${ }^{4}$ We reasoned that this methodology may be exploited to generate $S$-glycosyl primary sulfonamides (sugar- $\mathrm{SO}_{2} \mathrm{NH}_{2}$ ) if an appropriate sulfonamide protecting group (R) could be employed throughout the synthesis. Our general approach, outlined in Scheme 1, consists of first introducing a thioacetate group at the anomeric centre of the per-O-acetylated sugar derivative. From this follows formation of a glycosyl sulfenamide (sugar-SNR 2 ), ${ }^{4 a}$ oxidation of the sulfenamide to give a glycosyl $\mathrm{N}$-protected sulfonamide (sugar- $\mathrm{SO}_{2} \mathrm{NR}_{2}$ ), and removal of the sulfonamide protecting (R) group to yield a primary sulfonamide at the anomeric centre (sugar$\mathrm{SO}_{2} \mathrm{NH}_{2}$ ). Thus the ' $\mathrm{R}$ ' protecting group must be readily introduced, be stable to oxidation conditions and readily removed following oxidation so as to unmask the primary sulfonamide group. As a final step the deprotection of the hydroxyls of the glycosyl moiety using standard Zemplen conditions ${ }^{5}$ should give the fully deprotected target $S$-glycosyl sulfonamide.

Scheme 1. Proposed strategy to synthesize $S$-glycosyl sulfonamides ${ }^{a, b}$

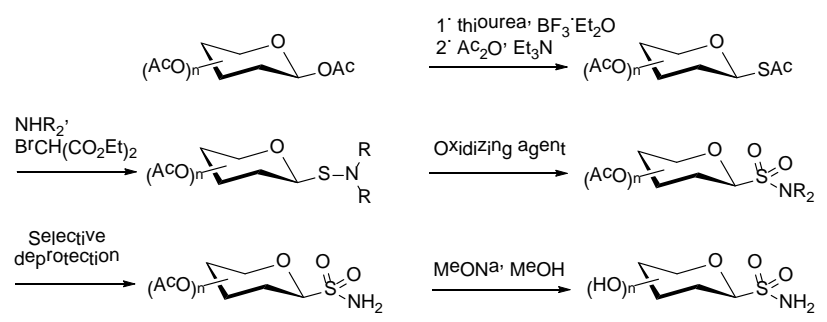

$a \mathrm{R}=$ sulfonamide protecting group.

$b$ This general strategy is illustrated for a monosaccharide but is proposed to apply to a range of carbohydrate substrates.

Given the prominence of the sulfonamide group in medicinal chemistry there is a surprising paucity in the literature with regard to protecting group strategies for primary sulfonamides. A literature search revealed a small number of examples where the 2,4-dimethoxybenzyl (DMB) substituent had been employed as a protecting group for sulfonamides. ${ }^{6}$ We commenced investigation of the DMB moiety as a protecting group with the synthesis of the $N, N-(\mathrm{DMB})_{2}$ glucosyl sulfenamide $2 \mathbf{a}$. Compound 2a was 
prepared with high anomeric stereoselectivity from 1, diethyl bromomalonate and bis(2,4dimethoxybenzyl)amine as base, Scheme 2, route a. ${ }^{4 a}$ Oxidation of 2a with $m$-CPBA led to a complex mixture of components and we postulated that the byproduct (m-chlorobenzoic acid) of the reaction partially cleaves the acid labile DMB groups. ${ }^{7}$ Misra recently reported the rapid oxidation of thioglycosides to glycosyl sulfones using a combination of $\mathrm{KMnO}_{4}$ and $\mathrm{CuSO}_{4} .5 \mathrm{H}_{2} \mathrm{O}$ in acetonitrile and water. ${ }^{8}$ These oxidative conditions are neutral and Misra confirmed compatibility with several acid labile hydroxyl protecting groups of sugar derivatives including benzylidene acetal, isopropylidene acetal and TBDMS. ${ }^{8}$ We were thus encouraged to investigate the capacity of this mild oxidative protocol to effect the oxidation of sulfenamide $\mathbf{2 a}$ in the presence of our acid labile protecting group. Gratifyingly the oxidation proceeded smoothly leading to the $\mathrm{N}, \mathrm{N}-(\mathrm{DMB})_{2}$ protected glucosyl sulfonamide 3a, Scheme 2, route a. Next the DMB groups of 3a were removed using acidic conditions (10\% TFA in $\mathrm{CH}_{2} \mathrm{Cl}_{2}$ ) and this reaction was complete after $2 \mathrm{~h}$ and delivered the target per-O-acetylated glucosyl sulfonamide 4. Zemplen's conditions ${ }^{5}$ were employed to remove the acetate groups of $\mathbf{4}$ to prepare the fully deprotected anomeric sulfonamide $\mathbf{5}$. Sulfonamides $\mathbf{4}$ and $\mathbf{5}$ were spectroscopically characterized using 1D and 2D NMR $\left({ }^{1} \mathrm{H},{ }^{13} \mathrm{C}\right.$, gCOSY, gHSQC) and ESI HRMS. All characterization data were consistent with the target structures. A characteristic signal for the sulfonamide protons $\left(\mathrm{SO}_{2} \mathrm{NH}_{2}\right)$ of $\mathbf{4}$ was observed at $\delta 5.01 \mathrm{ppm}$ in $\mathrm{CDCl}_{3}$ and $\delta 7.22 \mathrm{ppm}$ in $d^{6}$-DMSO and for $\mathbf{5} \delta 6.69 \mathrm{ppm}$ in $d^{6}$-DMSO.

Although we had successfully synthesized the S-glucosyl primary sulfonamides $\mathbf{4}$ and $\mathbf{5}$ we sought to further improve our synthesis. The $N, N$-(DMB $)_{2}$ protected compounds - sulfenamide 2 a and sulfonamide 3a - proved highly sensitive on normal phase silica and were difficult to manipulate without substantial degradation during standard purification protocols. Even when the silica was preconditioned with $1 \% \mathrm{Et}_{3} \mathrm{~N}$ degradation occurred (including loss of the DMB group). Further to this bis(2,4-dimethoxybenzyl)amine is not commercially available and required a two step synthesis over two days using hazardous reagents. We therefore decided to investigate the utility of the primary amine 
derivatives 2,4-dimethoxybenzylamine and 4-methoxybenzylamine in our synthesis as these have the advantage of being readily available from commercial suppliers. As primary amines we had a concern that these reagents may cause undesirable $O$-deacetylation of the carbohydrate moiety, ${ }^{4 a}$ however this was not observed. As a protecting group, the 4-methoxybenzyl substituent (PMB) should have intermediate stability towards acid promoted removal compared to the more acid labile DMB protecting group.

Scheme 2. Synthesis of sulfonamides 4 and 5 from per- $O$-acetylated D-glucose
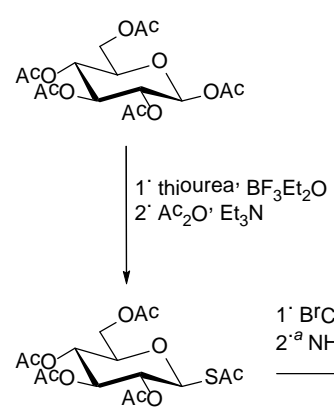

1
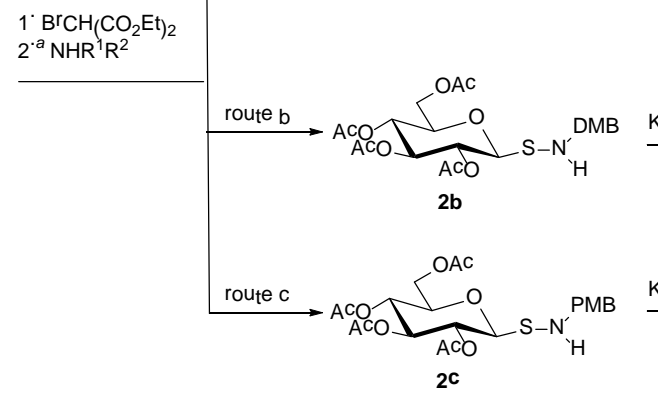
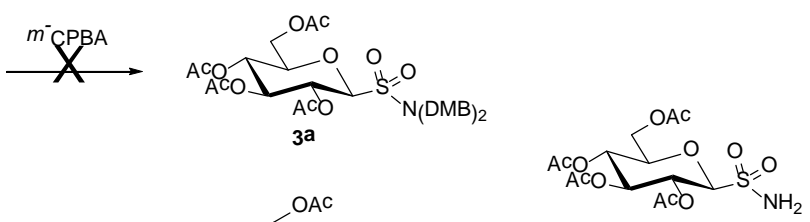

$\mathrm{KMnO}_{4} / \mathrm{CuSO}_{4}$
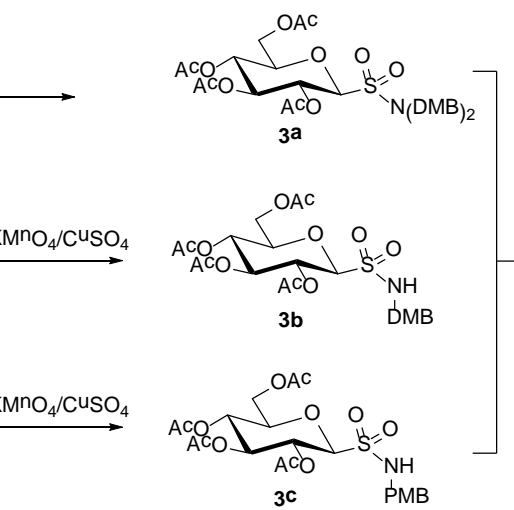

$3 c$

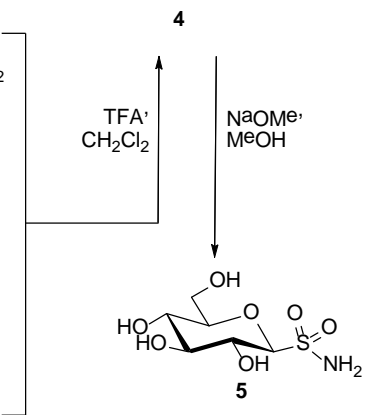

${ }^{a}$ route a: $\mathrm{R}^{1}=\mathrm{R}^{2}=\mathrm{DMB}$; route $\mathrm{b}: \mathrm{R}^{1}=\mathrm{DMB}, \mathrm{R}^{2}=\mathrm{H}$; route c: $\mathrm{R}^{1}=\mathrm{PMB}, \mathrm{R}^{2}=\mathrm{H}$

Sulfenamides 2b (NH-DMB) and 2c (NH-PMB) were synthesized from 1 similarly to 2a, Scheme 2, routes b and c, respectively. As observed for $\mathbf{2 a}$, compound $\mathbf{2} \mathbf{b}$ was also unstable on normal phase silica preconditioned with $1 \% \mathrm{Et}_{3} \mathrm{~N}$ and so was instead semi-purified on $\mathrm{C} 18$ reverse phase silica to remove excess amine reagent. The PMB compound 2c was more stable to chromatography on normal phase silica (conditioned with $1 \% \mathrm{Et}_{3} \mathrm{~N}$ ) consistent with the reduced lability of this protecting group towards an acidic environment. Compounds $\mathbf{2 b}$ and $\mathbf{2 c}$ were oxidized to the sulfonamides $\mathbf{3 b}$ and $\mathbf{3 c}$, respectively, applying our previously used neutral oxidation conditions $\left(\mathrm{KMnO}_{4} / \mathrm{CuSO}_{4} \cdot 5 \mathrm{H}_{2} \mathrm{O}\right)$. A small 
amount of protecting group loss was observed during these reactions however oxidation predominates leading to a relatively clean reaction mixture that required only an aqueous workup followed by purification on reserve phase silica (C18). The deprotection of $\mathbf{3 b}$ and $\mathbf{3 c}$ to remove the DMB and PMB groups was then achieved by stirring with a solution of TFA in $\mathrm{CH}_{2} \mathrm{Cl}_{2}$ at room temperature to generate the primary sulfonamide 4 . Deprotection of $\mathbf{3 b}$ was complete in 2 h (25\% TFA, yield 10\% over three steps from thioacetate 1) while deprotection of 3c required 2 days (50\% TFA, yield 26\% over three steps from thioacetate $\mathbf{1})$.

Each of the three sulfonamide protecting groups: (DMB) $)_{2}, \mathrm{DMB}$ and $\mathrm{PMB}$ allowed the successful transformation of thioacetate $\mathbf{1}$ to the primary sulfonamide $\mathbf{4}$ through the combination of neutral oxidizing conditions followed by acid catalyzed selective deprotection. The ready availability of the starting amines for routes $\mathrm{b}$ and $\mathrm{c}$ is an advantage, however the ease of removal of the DMB group compared to the PMB group brings us to conclude that DMB (route b) is the most practical protecting group to facilitate the synthesis of anomeric sulfonamides for our substrates. Although not strictly a onepot synthesis the use of intermediates $\mathbf{2} \mathbf{b}$ and $\mathbf{3 b}$ in a semi-purified form following rapid chromatography on reverse phase silica (C18 SPE cartridges) proved highly effective and made the overall transformation of glycosyl thioacetate to anomeric sulfonamide a relatively succinct synthetic procedure.

Having the reaction conditions to form anomeric primary sulfonamides established on the D-glucose moiety (to prepare 4 and 5) we then applied our methodology to a variety of mono- (D-galactose, Dglucuronic acid) and disaccharide (maltose, lactose) derivatives, Table 1. Glycosyl thioacetates 6-9 were synthesized from per-O-acetylated sugars using thiourea and boron trifluoro etherate in yields ranging from 47-79\%. ${ }^{9}$ The corresponding sulfenamides were prepared from 2,4-dimethoxybenzyl amine similarly to $\mathbf{2 b}$ and were used in the next step following semi-purification on reverse phase silica (C18). Oxidation of sulfenamides as before $\left(\mathrm{KMnO}_{4} / \mathrm{CuSO}_{4} .5 \mathrm{H}_{2} \mathrm{O}\right)$ followed by deprotection of the DMB 
group with 25\% TFA in $\mathrm{CH}_{2} \mathrm{Cl}_{2}$ for $2 \mathrm{~h}$ gave the target anomeric primary sulfonamides $\mathbf{1 0 - 1 3}$ (yields 18-45\% over three steps). Next Zemplen's conditions ${ }^{5}$ were applied to remove the acetate groups of compounds 10, 12 and 13, while $\mathrm{NaOH}(0.07 \mathrm{M})$ was used to deprotect both the hydroxyl and carboxylic acid groups of the glucuronic acid derivative 11. The fully deprotected anomeric sulfonamide compounds 14-17 were obtained in good to high yields, Table 1.

Table 1. Synthesized S-glycosyl sulfonamides

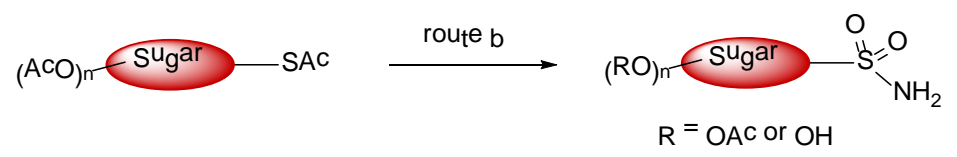

\begin{tabular}{lll}
\hline Thioacetate & Compound & Yield
\end{tabular}

(\%)

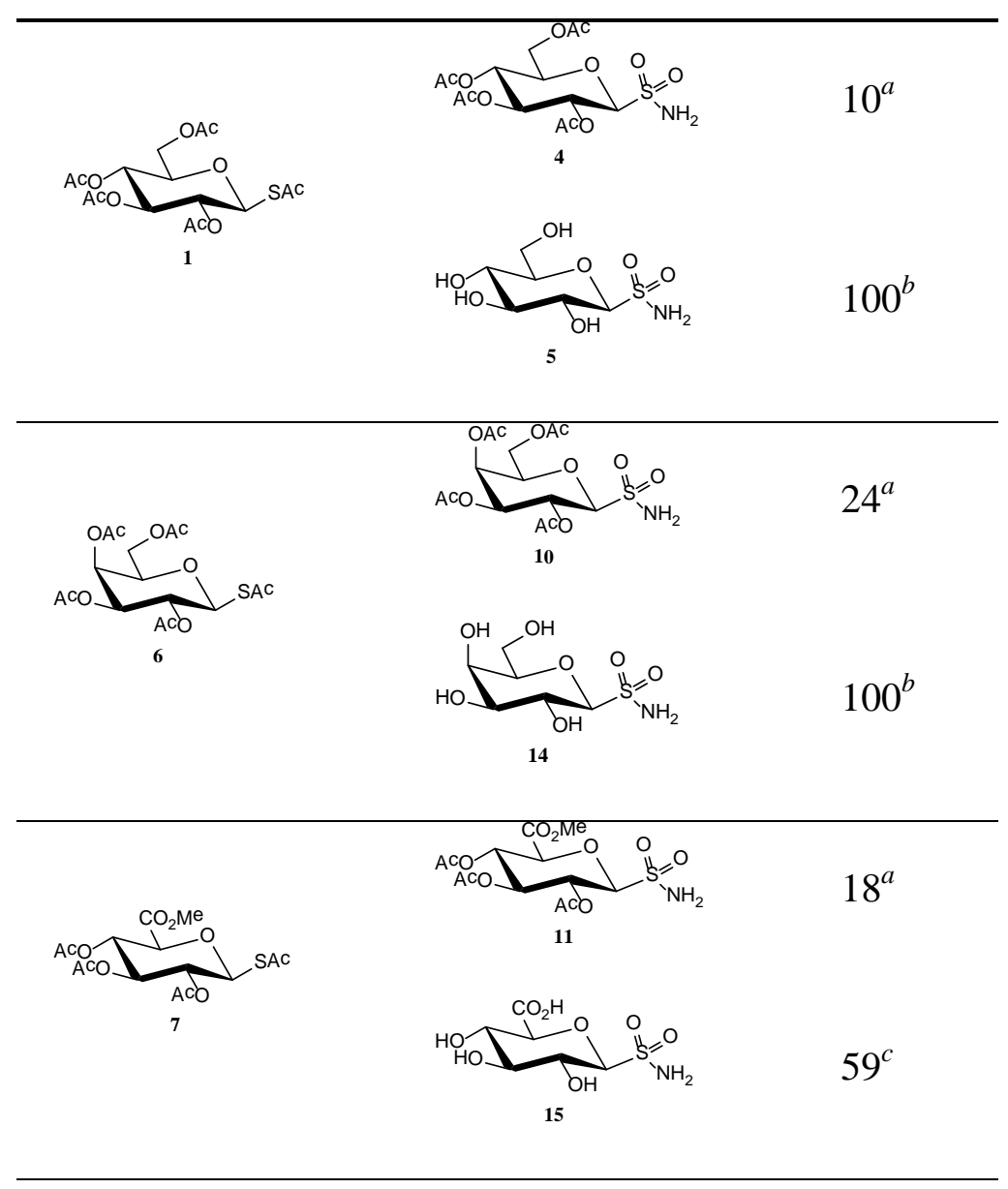



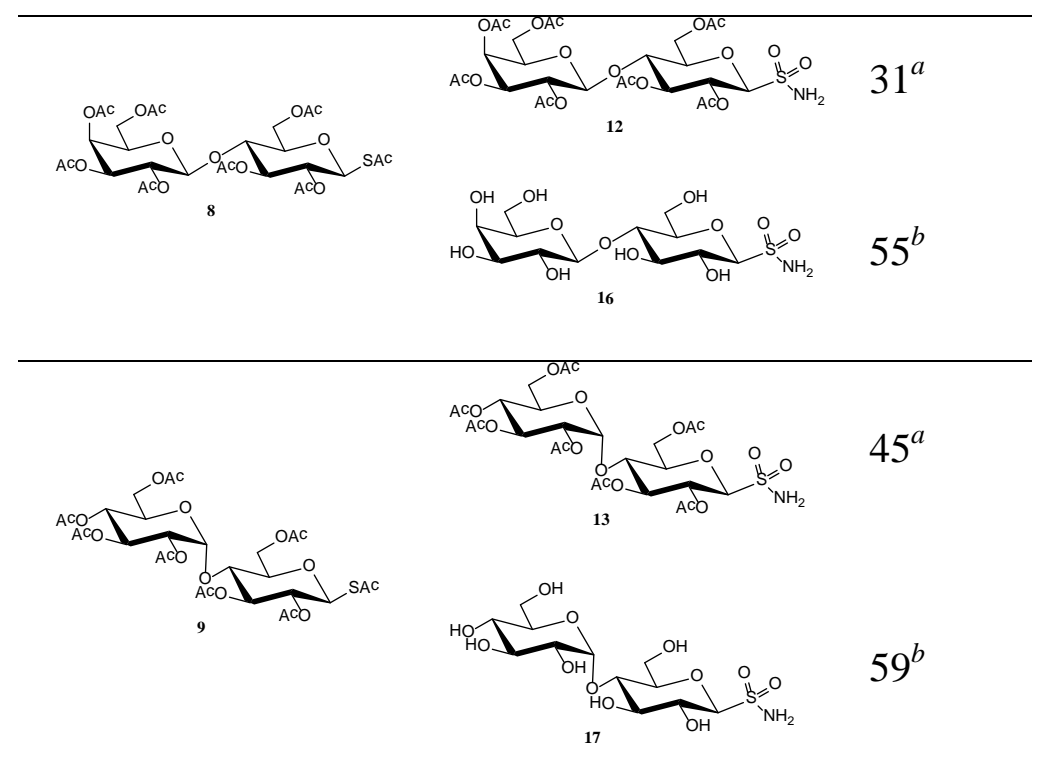

${ }^{a}$ \%Yield over three steps from thioacetate.

${ }^{b} \%$ Yield for Zemplen deprotection.

${ }^{c}$ \%Yield for deprotection using sodium hydroxide.

\section{Conclusions}

In conclusion we have developed a relatively simple and general procedure for the synthesis of $S$ glycosyl primary sulfonamides starting from per-O-acetylated sugar derivatives. Our approach has been to optimize reaction transformations appropriate for the protecting group requirements leading to the anomeric sulfonamide. We also propose that the DMB group is a viable sulfonamide protecting group provided neutral reaction conditions are employed.

\section{Experimental}

\section{$N, N$-bis(2,4-dimethoxybenzyl)-1-S-(2,3,4,6- tetra-O-acetyl)-D-glucopyranosylsulfenamide (2a).}

To a solution of thioacetate derivative 1 (1.21 g, $2.98 \mathrm{mmol}, 1.0$ equiv.) in anhydrous $\mathrm{MeOH}(60 \mathrm{~mL})$ under argon was added diethylbromomalonate $(1.2 \mathrm{~mL}, 7.13 \mathrm{mmol}, 2.4$ equiv.). The solution was stirred at $\mathrm{rt}$ for $20 \mathrm{~min}$ and then bis(2,4-dimethoxybenzyl)amine (3.79 g, $11.9 \mathrm{mmol}, 4.0$ equiv.) added. The reaction mixture was stirred overnight at rt after which a precipitate was formed. The precipitate was 
collected by filtration and washed with $\mathrm{MeOH}$ to afford the title compound 2a as a white solid (1.60 g, 2.35 mmol, 79\%). $\mathrm{R}_{f}=0.47$ (1:1 EtOAc-hexane). $\mathrm{mp}=143-144{ }^{\circ} \mathrm{C} .{ }^{1} \mathrm{H}$ NMR (500 MHz, DMSO): $\delta=$ 7.16 (d, $\left.J=8.5 \mathrm{~Hz}, 2 \mathrm{H}, \mathrm{H}_{\text {arom. }}\right), 6.48$ (m, 4H, $\mathrm{H}_{\text {arom.) }}$ ) 5.32 (t, $\left.J=9.5 \mathrm{~Hz}, 1 \mathrm{H}, \mathrm{H}-3\right), 5.21$ (d, $J=10.0 \mathrm{~Hz}$, 1H, H-1), 4.86 (t, $J=9.5 \mathrm{~Hz}, 1 \mathrm{H}, \mathrm{H}-4), 4.65$ (t, $J=10.0 \mathrm{~Hz}, 1 \mathrm{H}, \mathrm{H}-2), 4.14$ (dd, $J=6.0,12.5 \mathrm{~Hz}, 1 \mathrm{H}, \mathrm{H}-$ 6a), 4.06 (m, 1H, H-5), 4.02 (m, 1H, H-6b), 3.99 (m, 4H, NCH $\mathrm{N}_{2}$, 3.74, 3.70 (2 × s, 12H, OCH $), 2.01$, 1.97, 1.94, $1.83\left(4 \times \mathrm{s}, 12 \mathrm{H}, \mathrm{OCOCH}_{3}\right)$, assignments were confirmed by ${ }^{1} \mathrm{H}-{ }^{1} \mathrm{H}$ gCOSY. LRMS $\left(\mathrm{ESI}^{+}\right)$: $m / z=680[\mathrm{M}+\mathrm{H}]^{+}, 702[\mathrm{M}+\mathrm{Na}]^{+}$.

\section{$N$-(2,4-dimethoxybenzyl)-1-S-(2,3,4,6- tetra-O-acetyl)-D-glucopyranosylsulfenamide (2b). The} title compound was synthesized from thioacetate 1 (300 mg, 0.74 mmol, 1.0 equiv.), diethylbromomalonate ( $0.30 \mathrm{~mL}, 1.78 \mathrm{mmol}, 2.4$ equiv.) and 2,4-dimethoxybenzylamine (0.42 mL, 2.80 mmol, 3.8 equiv.) using the procedure described for compound 2a. The crude product was semi-purified on reverse phase silica (C-18 pre-packed cartridge, 5 g sorbent) with a gradient of $\mathrm{H}_{2} \mathrm{O}-\mathrm{MeOH}$. The title compound eluted over four fractions (40 - 70\% MeOH) and was obtained as a yellow oil (265 mg, 0.50 mmol, 68\%). $\mathrm{R}_{f}=0.57$ (1:1 EtOAc-hexane). ${ }^{1} \mathrm{H}$ NMR (500 MHz, DMSO): $\delta=7.13(\mathrm{~d}, J=8.0 \mathrm{~Hz}, 1 \mathrm{H}$, $\mathrm{H}_{\text {arom.) }}$ ) 6.53 (d, $J=2.5 \mathrm{~Hz}, 1 \mathrm{H}, \mathrm{H}_{\text {arom. }}$ ), 6.46 (dd, $J=2.5,8.5 \mathrm{~Hz}, 1 \mathrm{H}, \mathrm{H}_{\text {arom. }}$ ), 5.35 (t, $J=9.5 \mathrm{~Hz}, 1 \mathrm{H}, \mathrm{H}-$ 3), 5.00 (t, $J=10.0 \mathrm{~Hz}, 1 \mathrm{H}, \mathrm{H}-2), 4.89$ (t, $J=9.5 \mathrm{~Hz}, 1 \mathrm{H}, \mathrm{H}-4), 4.69$ (t, $J=10.0 \mathrm{~Hz}, 1 \mathrm{H}, \mathrm{H}-1), 4.06$ (m, 4H, H-6a, H-6b, $\mathrm{NCH}_{2}$ ), 3.98 (m, 1H, H-5), 3.79, 3.75 (2 × s, 6H, OCH $), 2.00,1.99,1.97,1.96(4 \times \mathrm{s}$, 12H, OCOCH$H_{3}$ ), assignments were confirmed by ${ }^{1} \mathrm{H}^{-1} \mathrm{H}$ gCOSY. LRMS $\left(\mathrm{ESI}^{+}\right): \mathrm{m} / \mathrm{z}=530[\mathrm{M}+\mathrm{H}]^{+}$, $552[\mathrm{M}+\mathrm{Na}]^{+}$.

\section{$N, N$-bis(2,4-dimethoxybenzyl)-1-S-(2,3,4,6- tetra-O-acetyl)-D-glucopyranosylsulfonamide (3a). A}

finely ground mixture of the catalytic oxidative system $\left(\mathrm{KMnO}_{4}: \mathrm{CuSO}_{4} .5 \mathrm{H}_{2} \mathrm{O} ; 1: 1\right.$; w/w; $2.4 \mathrm{~g}, 6.3: 4.0$ equiv.) was dissolved in $\mathrm{H}_{2} \mathrm{O}(5 \mathrm{~mL})$ and added to a solution of sulfenamide 2a (825 mg, $1.21 \mathrm{mmol}$, 1.0 equiv.) in acetonitrile $(15 \mathrm{~mL})$. The reaction was stirred at $\mathrm{rt}$ for $1.5 \mathrm{~h}$ after which the acetonitrile 
was evaporated under reduced pressure and the remaining aqueous residue extracted with EtOAc $(\times 1)$.

The organic phase was washed with brine $(\times 2)$ and aqueous extracts back extracted with EtOAc $(\times 2)$. The organic extracts were combined, dried over $\mathrm{MgSO}_{4}$, filtered and concentrated in vacuo. The residue was purified by flash chromatography (2:3 EtOAc-hexane, $\left.1 \% \mathrm{Et}_{3} \mathrm{~N}\right)$ affording the title compound as a yellow oil (150 mg, $0.21 \mathrm{mmol}, 17 \%) . \mathrm{R}_{f}=0.32$ (1:1 EtOAc-hexane). ${ }^{1} \mathrm{H}$ NMR (500 MHz, $\left.\mathrm{CDCl}_{3}\right): \delta=$ 7.18 (m, 2H, $\left.\mathrm{H}_{\text {arom.) }}\right) 6.38$ (dd, $\left.J=2.5,8.0 \mathrm{~Hz}, 2 \mathrm{H}, \mathrm{H}_{\text {arom. }}\right), 6.35$ (d, $J=2.5 \mathrm{~Hz}, 2 \mathrm{H}, \mathrm{H}_{\text {arom. }}$ ), 5.34 (t, $J=$ $9.5 \mathrm{~Hz}, 1 \mathrm{H}, \mathrm{H}-2), 5.13$ (t, $J=9.5 \mathrm{~Hz}, 1 \mathrm{H}, \mathrm{H}-3), 4.97$ (t, $J=10.0 \mathrm{~Hz}, 1 \mathrm{H}, \mathrm{H}-4), 4.51$ (d, $J=16.0 \mathrm{~Hz}, 2 \mathrm{H}$, $\mathrm{NCH}_{2}$ ), 4.29 (d, $J=10.0 \mathrm{~Hz}, 1 \mathrm{H}, \mathrm{H}-1$ ), 4.20 (d, $J=16.0 \mathrm{~Hz}, 4 \mathrm{H}, \mathrm{NCH}_{2}$ ), 3.99 (dd, $J=5.5,10.0 \mathrm{~Hz}, 1 \mathrm{H}$, H-6a), 3.94 (dd, $J$ = 2.5, $10.0 \mathrm{~Hz}, 1 \mathrm{H}, \mathrm{H}-6 \mathrm{~b}), 3.74,3.69$ (2 × s, 12H, OCH $\mathrm{OCH}_{3}, 3.53$ (m, 1H, H-5), 2.00, 1.95, 1.94, $1.93(4 \times \mathrm{s}, 12 \mathrm{H}, \mathrm{OCOCH})_{3}$, assignments were confirmed by ${ }^{1} \mathrm{H}-{ }^{1} \mathrm{H}$ gCOSY. LRMS $\left(\mathrm{ESI}^{+}\right)$: $m / z=734[\mathrm{M}+\mathrm{Na}]^{+}$. HRMS (ESI) calcd for $\mathrm{C}_{32} \mathrm{H}_{41} \mathrm{~N}_{1} \mathrm{O}_{15} \mathrm{SNa}^{+}:$734.2089, Found: 734.2085.

$N$-(2,4-dimethoxybenzyl)-1-S-(2,3,4,6- tetra-O-acetyl)-D-glucopyranosylsulfonamide (3b). The title compound was synthesized from sulfenamide $2 \mathbf{b}$ ( $3.54 \mathrm{~g}, 6.68 \mathrm{mmol}, 1.0$ equiv.) with the procedure described for compound 3a. After washing, the crude yellow oil obtained was used without purification. $\mathrm{R}_{f}=0.31$ (1:1 EtOAc-hexane). LRMS $\left(\mathrm{ESI}^{+}\right) \mathrm{m} / \mathrm{z} 584[\mathrm{M}+\mathrm{Na}]^{+}$. HRMS (ESI) calcd for $\mathrm{C}_{23} \mathrm{H}_{31} \mathrm{~N}_{1} \mathrm{O}_{13} \mathrm{SNa}^{+}:$584.1408, Found: 584.1411.

\section{$N$-(4-methoxybenzyl)-1-S-(2,3,4,6- tetra-O-acetyl)-D-glucopyranosylsulfonamide $\quad$ (3c). To a} solution of thioacetate derivative $\mathbf{1}$ (307 mg; $0.76 \mathrm{mmol}$, 1.0 equiv.) in anhydrous $\mathrm{MeOH}$ (15 mL) under argon was added diethylbromomalonate $(0.3 \mathrm{~mL}, 1.78 \mathrm{mmol}, 2.3$ equiv.). The solution was stirred at $\mathrm{rt}$ for $20 \mathrm{~min}$ and then 4-methoxybenzylamine $(0.50 \mathrm{~mL}, 3.85 \mathrm{mmol}$, 5.1 equiv.) added. The reaction mixture was stirred overnight at rt. The mixture was concentrated, then purified by flash chromatography (2:3 EtOAc-hexane, $\left.\mathrm{R}_{f}=0.38\right)$. A yellow oil $(313 \mathrm{mg}, 0.63 \mathrm{mmol}, 83 \%)$ was obtained. Oxidation of the sulfenamide occurred as described for the preparation of compound 3a using catalytic 
system ( $\mathrm{KMnO}_{4}: \mathrm{CuSO}_{4} .5 \mathrm{H}_{2} \mathrm{O} ; 1: 1 ; \mathrm{w} / \mathrm{w} ; 1.0$ g, 4.2:2.6 equiv.) in $\mathrm{CH}_{3} \mathrm{CN}-\mathrm{H}_{2} \mathrm{O}$ (5:1, $\left.180 \mathrm{~mL}\right)$. Crude mixture was semi-purified on reverse phase silica (C-18 pre-packed cartridge, 5 g sorbent) eluted with a gradient of $\mathrm{H}_{2} \mathrm{O}-\mathrm{MeOH}$. Elution of sulfonamide 3c occurred over five fractions (60 - 100\% MeOH). $\mathrm{R}_{f}$ $=0.37\left(1: 1\right.$ EtOAc-hexane). LRMS $\left(\mathrm{ESI}^{+}\right) \mathrm{m} / \mathrm{z} 554[\mathrm{M}+\mathrm{Na}]^{+}$.

1-S-(2,3,4,6- tetra-O-acetyl)-D-glucopyranosylsulfonamide (4). The crude mixture of the protected sulfonamide $3 \mathbf{b}$ was dissolved in a solution of $25 \%$ trifluoroacetic acid (TFA) in $\mathrm{CH}_{2} \mathrm{Cl}_{2}(24 \mathrm{~mL})$ and stirred $2 \mathrm{~h}$ at rt. Solvent and TFA were evaporated under reduced pressure and the remaining residue diluted with $\mathrm{CH}_{2} \mathrm{Cl}_{2}(150 \mathrm{~mL})$ and washed with brine $(\times 3)$. The aqueous extracts were back extracted with $\mathrm{CH}_{2} \mathrm{Cl}_{2}(\times 2)$ and then all organic extracts were combined, dried over $\mathrm{MgSO}_{4}$, filtered and evaporated. The residue was purified by flash chromatography with solid addition (99:1 $\mathrm{CH}_{2} \mathrm{Cl}_{2}-$ $\mathrm{MeOH}$ ) to afford the title sulfonamide 4 as a colorless solid (372 mg, $0.90 \mathrm{mmol}$, 14\% over two steps from 2b). $\mathrm{R}_{f}=0.26$ (1:1 EtOAc-hexane). $\mathrm{mp}=210-211^{\circ} \mathrm{C} .[\alpha]_{\mathrm{D}}^{25}=-6(c=1.0$, chloroform $) .{ }^{1} \mathrm{H}$ NMR (500 MHz, $\left.\mathrm{CDCl}_{3}\right): \delta=5.36$ (d, $\left.J=9.5 \mathrm{~Hz}, 1 \mathrm{H}, \mathrm{H}-3\right), 5.33$ (d, $\left.J=9.5 \mathrm{~Hz}, 1 \mathrm{H}, \mathrm{H}-2\right), 5.14$ (t, $J=9.5 \mathrm{~Hz}$, 1H, H-4), 4.96 (br s, 2H, NH2), 4.38 (d, $J=9.0 \mathrm{~Hz}, 1 \mathrm{H}, \mathrm{H}-1$ ), 4.37 (dd, $J=5.0,12.5 \mathrm{~Hz}, 1 \mathrm{H}, \mathrm{H}-6 \mathrm{a}$ ), 4.18 (dd, $J=2.5,12.5 \mathrm{~Hz}, 1 \mathrm{H}, \mathrm{H}-6 \mathrm{~b}), 3.87$ (ddd, $J=2.5,5.0,10.5,1 \mathrm{H}, \mathrm{H}-5), 2.11,2.10,2.06,2.04(4 \times \mathrm{s}$, $\left.12 \mathrm{H}, \mathrm{OCOCH}_{3}\right)$, assignments were confirmed by ${ }^{1} \mathrm{H}-{ }^{1} \mathrm{H}$ gCOSY. ${ }^{13} \mathrm{C}$ NMR $\left(125 \mathrm{MHz}, \mathrm{CDCl}_{3}\right): \delta=$ 170.9, 170.6, 169.9, $169.4\left(\mathrm{OCOCH}_{3}\right), 87.5$ (C-1), 76.7 (C-5), 72.7 (C-3), 68.2 (C-2), 67.9 (C-4), 61.6 (C-6), 20.7, 20.6, 20.5 (2C) $\left(\mathrm{OCOCH}_{3}\right)$, assignments were confirmed by ${ }^{1} \mathrm{H}^{-13} \mathrm{C}$ HSQC. LRMS $\left(\mathrm{ESI}^{+}\right)$: $m / z=434[\mathrm{M}+\mathrm{Na}]^{+} ;$LRMS $\left(\mathrm{ESI}^{-}\right): m / z=410[\mathrm{M}-\mathrm{H}]^{-}$. HRMS (ESI) calcd for $\mathrm{C}_{14} \mathrm{H}_{21} \mathrm{~N}_{1} \mathrm{O}_{11} \mathrm{SNa}^{+}$: 434.0728, Found: 434.0708.

1-S-D-glucopyranosylsulfonamide (5). General Procedure 1. Fully deprotected compound 5 was prepared by treating a solution of the per- $O$-acetylated compound 4 (191 mg, $0.46 \mathrm{mmol}, 1.0$ equiv.) in anhydrous $\mathrm{MeOH}(15 \mathrm{~mL})$ at $0{ }^{\circ} \mathrm{C}$ with methanolic sodium methoxide (25\% w/v, $1 \mathrm{~mL}, 1.0$ equiv.), 
final $\mathrm{pH}=14$. The reaction was warmed to rt and left to stir until full deprotection was evident by TLC ( $\sim 2$ h). The solution was neutralized with Amberlite IR-120 $\left[\mathrm{H}^{+}\right]$, filtered and the resin washed several times with methanol. The solvent was evaporated under reduced pressure and lyophilized to dryness to furnish the title sulfonamide 5 as a slightly yellow hygroscopic solid (113 mg, 0.46 mmol, 100\%) which was pure by ${ }^{1} \mathrm{H}$ NMR. $\mathrm{R}_{f}=0.1\left(9: 1 \mathrm{CH}_{3} \mathrm{CN}-\mathrm{H}_{2} \mathrm{O}\right) . \mathrm{mp}=63-64{ }^{\circ} \mathrm{C} .[\alpha]^{25}{ }_{\mathrm{D}}=+5(c=0.9, \mathrm{MeOH})$. ${ }^{1} \mathrm{H}$ NMR (500 MHz, DMSO): $\delta=6.69$ (br s, 2H, NH ), $5.11(\mathrm{~m}, 1 \mathrm{H}, \mathrm{OH}), 5.05$ (d, $J=5.0 \mathrm{~Hz}, 1 \mathrm{H}, \mathrm{OH}$ ), 5.02 (d, $J=5.5 \mathrm{~Hz}, 1 \mathrm{H}, \mathrm{OH}), 4.47$ (m, 1H, OH), 4.05 (d, $J=9.5 \mathrm{~Hz}, \mathrm{H}-1), 3.68$ (m, 1H, H-6a), 3.45 (m, 2H, H-6b, H-2), 3.25 (m, 2H, H-5, H-3), 3.05 (m, 1H, H-4), assignments were confirmed by ${ }^{1} \mathrm{H}-{ }^{1} \mathrm{H}$ gCOSY. ${ }^{13} \mathrm{C}$ NMR (125 MHz, DMSO): $\delta=90.5$ (C-1), 81.3 (C-5), 77.3 (C-3), 70.6 (C-2), 69.6 (C-4), 61.2 (C-6) assignments were confirmed by ${ }^{1} \mathrm{H}^{-13} \mathrm{C}$ HSQC. LRMS (ESI $\left.{ }^{+}\right): m / z=266[\mathrm{M}+\mathrm{Na}]^{+}$. HRMS (ESI) calcd for $\mathrm{C}_{6} \mathrm{H}_{13} \mathrm{~N}_{1} \mathrm{O}_{7} \mathrm{SNa}^{+}$: 266.0305, Found: 266.0309.

1-S-(2,3,4,6- tetra-O-acetyl)-D-galactopyranosylsulfonamide (10). General Procedure 2. The title compound was synthesized over three steps from thioacetate derivative $\mathbf{6}$. First, the intermediate sulfenamide was prepared from thioacetate 6 (4.00 g, $9.84 \mathrm{mmol}, 1.0$ equiv.), diethylbromomalonate (0.30 mL, $1.78 \mathrm{mmol}, 2.4$ equiv.) and 2,4-dimethoxybenzylamine (0.42 mL, $2.80 \mathrm{mmol}, 3.8$ equiv.) using the procedure described for the preparation of compound 2a. This intermediate was semi-purified on reverse phase silica (C-18 pre-packed cartridge, 5 g sorbent) using a gradient of $\mathrm{H}_{2} \mathrm{O}-\mathrm{MeOH}$ and eluted over three fractions $(60-80 \% \mathrm{MeOH})$ to give a yellow oil. This sulfenamide intermediate was immediately submitted to oxidation using the procedure described for preparation of compound 3a to afford the $N$-protected sulfonamide intermediate which was used without purification. $\mathrm{R}_{f}=0.29$ (1:1 EtOAc-hexane). Removal of the DMB protecting group was achieved as described for compound 4. The yellow oil obtained was purified by flash chromatography (1:1 EtOAc-hexane) to afford the title sulfonamide 10 (980 mg, 2.38 mmol, 24\% over three steps). $\mathrm{R}_{f}=0.22$ (1:1 EtOAc-hexane). $[\alpha]^{25}=+5$ (c = 1.1, chloroform). ${ }^{1} \mathrm{H}$ NMR $\left(500 \mathrm{MHz}, \mathrm{CDCl}_{3}\right): \delta=5.50(\mathrm{t}, J=9.5 \mathrm{~Hz}, 1 \mathrm{H}, \mathrm{H}-2), 5.49(\mathrm{t}, J=9.5 \mathrm{~Hz}$, 1H, H-3), 5.19 (dd, $J=3.0,10.0 \mathrm{~Hz}, 1 \mathrm{H}, \mathrm{H}-4), 5.03$ (br s, 2H, NH ), 4.39 (d, $J=10.0 \mathrm{~Hz}, 1 \mathrm{H}, \mathrm{H}-1$ ), 
4.24 (m, 1H, H-6a), 4.16 (m, 1H, H-5), 4.11 (m, 1H, H-6b), 2.19, 2.11, 2.07, $2.02(4 \times \mathrm{s}, 12 \mathrm{H}$,

$\left.\mathrm{OCOCH}_{3}\right)$, assignments were confirmed by ${ }^{1} \mathrm{H}-{ }^{1} \mathrm{H}$ gCOSY. ${ }^{13} \mathrm{C} \mathrm{NMR}\left(125 \mathrm{MHz}, \mathrm{CDCl}_{3}\right): \delta=171.3$, 170.5, 170.1, $169.9\left(\mathrm{OCOCH}_{3}\right), 88.1$ (C-1), 75.6 (C-5), 70.8 (C-4), 67.1 (C-3), 65.6 (C-2), 61.1 (C-6), 20.7, 20.5 (2C), $20.4\left(\mathrm{OCOCH}_{3}\right)$, assignments were confirmed by ${ }^{1} \mathrm{H}^{-13} \mathrm{C}$ HSQC. LRMS $\left(\mathrm{ESI}^{+}\right): \mathrm{m} / \mathrm{z}=$ $434[\mathrm{M}+\mathrm{Na}]^{+}$; LRMS (ESI'): $\mathrm{m} / \mathrm{z}=410[\mathrm{M}-\mathrm{H}]^{+}$. HRMS (ESI) calcd for $\mathrm{C}_{14} \mathrm{H}_{21} \mathrm{~N}_{1} \mathrm{O}_{11} \mathrm{SNa}^{+}$: 434.0728, Found: 434.0706.

Methyl 1-S-(2,3,4,6- tetra-O-acetyl)-D-glucopyranuronylsulfonamide (11). The title compound was obtained from thioacetate 7 (2.94 g, $7.49 \mathrm{mmol}, 1.0$ equiv.) as described in general procedure 2 and obtained as a light yellow solid (542 mg, $1.36 \mathrm{mmol}, 18 \%$ over 3 steps). $\mathrm{R}_{f}=0.41$ (2:1 EtOAc-hexane). $\mathrm{mp}=200-202{ }^{\circ} \mathrm{C}[\alpha]_{\mathrm{D}}^{25}=-13(c=0.8$, chloroform $) .{ }^{1} \mathrm{H}$ NMR $\left(500 \mathrm{MHz}, \mathrm{CDCl}_{3}\right): \delta=5.41(\mathrm{t}, J=9.5$ Hz, 1H, H-3), 5.35 (t, $J=9.5$ Hz, 1H, H-2), 5.24 (t, $J=9.5$ Hz, 1H, H-4), 5.13 (br s, 2H, NH $), 4.41$ (d, $J=10.0 \mathrm{~Hz}, 1 \mathrm{H}, \mathrm{H}-1), 4.18(\mathrm{~d}, J=9.5 \mathrm{~Hz}, 1 \mathrm{H}, \mathrm{H}-1), 3.77$ (s, 3H, OCH $), 2.10,2.05(2 \times \mathrm{s}, 9 \mathrm{H}$, $\mathrm{OCOCH}_{3}$ ), assignments were confirmed by ${ }^{1} \mathrm{H}-{ }^{1} \mathrm{H}$ GCOSY. ${ }^{13} \mathrm{C}$ NMR $\left(125 \mathrm{MHz}, \mathrm{CDCl}_{3}\right): \delta=171.1$, 169.8, 169.5, $166.7\left(\mathrm{OCOCH}_{3}\right), 87.1$ (C-1), 76.2 (C-5), 72.0 (C-3), 69.3 (C-4), 68.1 (C-2), 53.4 $\left(\mathrm{CO}_{2} \mathrm{CH}_{3}\right)$, 20.9, 20.7, $20.6\left(\mathrm{OCOCH}_{3}\right)$, assignments were confirmed by ${ }^{1} \mathrm{H}^{-}{ }^{13} \mathrm{C}$ HSQC. LRMS $\left(\mathrm{ESI}^{+}\right)$: $m / z=420[\mathrm{M}+\mathrm{Na}]^{+} ;$LRMS (ESI $): m / z=396[\mathrm{M}-\mathrm{H}]^{+}$. HRMS (ESI) calcd for $\mathrm{C}_{13} \mathrm{H}_{19} \mathrm{~N}_{1} \mathrm{O}_{11} \mathrm{SNa}^{+}$: 420.0571, Found: 420.0580.

\section{$(2,3,4,6$-Tetra- $O$-acetyl- $\beta$-D-galactopyranosyl)-( $1 \rightarrow 4)-1,2,3,6$-tetra- $O$-acetyl-1-thio- $\beta$-D-}

glucopyranosylsulfonamide (12). The title compound was obtained from thioacetate 8 (4.36 g, 6.28 mmol, 1.0 equiv.) as described in general procedure 2 and obtained as a light orange solid (1.35 g, 1.93 mmol, 31\%). $\mathrm{R}_{f}=0.23$ (3:2 EtOAc-hexane). $\mathrm{mp}=103-105{ }^{\circ} \mathrm{C} .[\alpha]^{25}{ }_{\mathrm{D}}=-12\left(c=1.1\right.$, chloroform). ${ }^{1} \mathrm{H}$ NMR (500 MHz, $\left.\mathrm{CDCl}_{3}\right): \delta=5.37$ (d, $J=3.0 \mathrm{~Hz}, 1 \mathrm{H}, \mathrm{H}-4$ '), 5.32 (t, $J=9.0 \mathrm{~Hz}, 1 \mathrm{H}, \mathrm{H}-3$ ), 5.27 (t, $J=$ 9.5 Hz, 1H, H-2), 5.12 (dd, $J=8.0,10.5$ Hz, 1H, H-2'), 4.98 (dd, J = 3.5, 10.5 Hz, 1H, H-3’), 4.96 (br s, 
2H, NH $H_{2}$ ), 4.54 (dd, $\left.J=1.5,12.5 \mathrm{~Hz}, 1 \mathrm{H}, \mathrm{H}-6 \mathrm{a}\right), 4.51$ (d, $J=7.5 \mathrm{~Hz}, 1 \mathrm{H}, \mathrm{H}-1^{\prime}$ ), 4.37 (d, $J=10.0 \mathrm{~Hz}$, 1H, H-1), 4.20 (dd, $J=4.0,12.5$ Hz, 1H, H-6b), 4.16 (dd, $J=6.5,11.5$ Hz, 1H, H-6a'), 4.09 (dd, $J=$ 7.5, 11.0 Hz, 1H, H-6b'), 3.89 (m, 1H, H-5'), 3.86 (t, $J$ = 9.5 Hz, 1H, H-4), 3.79 (m, 1H, H-5), 2.17, 2.14, $2.08(6 \mathrm{H}), 2.07,2.06,1.98\left(6 \times \mathrm{s}, 21 \mathrm{H}, \mathrm{OCOCH}_{3}\right)$, assignments were confirmed by ${ }^{1} \mathrm{H}-{ }^{1} \mathrm{H}$ gCOSY. ${ }^{13} \mathrm{C}$ NMR $\left(125 \mathrm{MHz}, \mathrm{CDCl}_{3}\right): \delta=170.9,170.7,170.6,170.3,170.2,169.7,169.2\left(\mathrm{OCOCH}_{3}\right), 101.2(\mathrm{C}-$ 1’), 87.6 (C-1), 77.6 (C-5), 75.7 (C-4), 72.8 (C-3), 71.1 (C-3’), 71.1 (C-5’), 69.3 (C-2'), 68.5 (C-2), 66.8 (C-4'), 61.7 (C-6), 61.0 (C-6'), 21.0, 20.9 (2C), 20.8 (2C), 20.7 (2C) $\left(\mathrm{OCOCH}_{3}\right)$, assignments were confirmed by ${ }^{1} \mathrm{H}^{13}{ }^{13} \mathrm{C}$ HSQC. LRMS $\left(\mathrm{ESI}^{+}\right): \mathrm{m} / \mathrm{z}=722[\mathrm{M}+\mathrm{Na}]^{+}$; LRMS $\left(\mathrm{ESI}^{-}\right): \mathrm{m} / \mathrm{z}=697[\mathrm{M}-\mathrm{H}]^{-}$. HRMS (ESI) calcd for $\mathrm{C}_{26} \mathrm{H}_{37} \mathrm{~N}_{1} \mathrm{O}_{19} \mathrm{SNa}^{+}:$722.1573, Found: 722.1541 .

\section{$(2,3,4,6$-Tetra- $O$-acetyl- $\alpha$-D-glucopyranosyl)-( $1 \rightarrow 4)-1,2,3,6$-tetra- $O$-acetyl-1-thio- $\beta$-D-}

glucopyranosylsulfonamide (13). The title compound was obtained from thioacetate 9 (3.89 g, 5.60 mmol, 1.0 equiv.) as described in general procedure 2 and obtained as a light orange solid (1.76 g, 2.52 mmol, 45\%). $\mathrm{R}_{f}=0.25$ (3:2 EtOAc-hexane). $\mathrm{mp}=96-98{ }^{\circ} \mathrm{C} .[\alpha]^{25}{ }_{\mathrm{D}}=+57\left(c=1.3\right.$, chloroform). ${ }^{1} \mathrm{H}$ NMR (500 MHz, CDCl 3 ): $\delta=5.41$ (t, $J=9.0 \mathrm{~Hz}, 1 \mathrm{H}, \mathrm{H}-3$ ), 5.41 (d, $J=3.5 \mathrm{~Hz}, 1 \mathrm{H}, \mathrm{H}-1^{\prime}$ ), 5.37 (t, $J=$ $10.0 \mathrm{~Hz}, 1 \mathrm{H}, \mathrm{H}-3$ '), 5.19 (t, $J=9.5 \mathrm{~Hz}, 1 \mathrm{H}, \mathrm{H}-2), 5.07$ (t, $J=10.0 \mathrm{~Hz}, 1 \mathrm{H}, \mathrm{H}-4$ '), 4.95 (br s, 2H, NH $_{2}$ ), 4.87 (dd, $J=4.0,10.5 \mathrm{~Hz}, 1 \mathrm{H}, \mathrm{H}-2^{\prime}$ ), 4.55 (dd, $J=2.5,12.5 \mathrm{~Hz}, 1 \mathrm{H}, \mathrm{H}-6 \mathrm{a}$ ), 4.43 (d, $J=9.5 \mathrm{~Hz}, 1 \mathrm{H}, \mathrm{H}-$ 1), 4.32 (dd, $J=4.5,12.5$ Hz, 1H, H-6b), 4.24 (dd, $J=4.0,12.5$ Hz, 1H, H-6a'), 4.08 (dd, $J=2.5,12.5$ Hz, 1H, H-6b'), 4.044 (t, J = 9.0 Hz, 1H, H-4), 3.96 (m, 1H, H-5'), 3.85 (m, 1H, H-5), 2.16, 2.11, 2.07, 2.06, 2.05, 2.04, $2.02\left(7 \times \mathrm{s}, 21 \mathrm{H}, \mathrm{OCOCH}_{3}\right)$, assignments were confirmed by ${ }^{1} \mathrm{H}-{ }^{1} \mathrm{H}$ gCOSY.${ }^{13} \mathrm{C}$ NMR (125 MHz, $\left.\mathrm{CDCl}_{3}\right): \delta=170.7$ (3C), 170.6, 170.0, 169.9, $169.5\left(\mathrm{OCOCH}_{3}\right), 96.0$ (C-1'), 87.3 (C-1), 77.1 (C-5), 75.0 (C-3), 72.6 (C-4), 70.2 (C-2’), 69.3 (C-3’), 68.9 (C-5’), 68.8 (C-2), 68.2 (C-4'), 62.4 (C-6), 61.7 (C-6'), 20.9 (2C), 20.7 (3C), 20.6 (2C) $\left(\mathrm{OCOCH}_{3}\right)$, assignments were confirmed by ${ }^{1} \mathrm{H}^{13}{ }^{13} \mathrm{HSQC}$. LRMS $\left(\mathrm{ESI}^{+}\right): m / z=722[\mathrm{M}+\mathrm{Na}]^{+}$; LRMS (ESI): $\mathrm{m} / \mathrm{z}=697[\mathrm{M}-\mathrm{H}]^{-}$. HRMS (ESI) calcd for $\mathrm{C}_{26} \mathrm{H}_{37} \mathrm{~N}_{1} \mathrm{O}_{19} \mathrm{SNa}^{+}:$722.1573, Found: 722.1541. 
1-S-D-galactopyranosylsulfonamide (14). The title compound was prepared from 10 (295 mg, 0.72 mmol) as described in general procedure 1 (except the reaction was maintained overnight at $50{ }^{\circ} \mathrm{C}$ ). The lyophilized compound was obtained as an orange hygroscopic solid (174 mg, $0.72 \mathrm{mmol}, \sim 100 \%)$. $\mathrm{R}_{f}=$ $0.46\left(8: 2 \mathrm{CH}_{3} \mathrm{CN}-\mathrm{H}_{2} \mathrm{O}\right) . \mathrm{mp}=58-59{ }^{\circ} \mathrm{C} .[\alpha]^{25}{ }_{\mathrm{D}}=+11(c=1.2, \mathrm{MeOH}) .{ }^{1} \mathrm{H}$ NMR $\left(400 \mathrm{MHz}, \mathrm{D}_{2} \mathrm{O}\right): \delta=$ 4.49 (d, $J=9.6 \mathrm{~Hz}, \mathrm{H}-1), 4.06$ (d, $J=3.2 \mathrm{~Hz}, 1 \mathrm{H}, \mathrm{H}-4), 4.04$ (t, $J=9.2 \mathrm{~Hz}, 1 \mathrm{H}, \mathrm{H}-2), 3.72-3.64$ (m, 3H, H-5, H-6a, H-6b), 3.62 (dd, $J=3.6,9.6 \mathrm{~Hz}, 1 \mathrm{H}, \mathrm{H}-3$ ) assignments were confirmed by ${ }^{1} \mathrm{H}-{ }^{1} \mathrm{H}$ gCOSY. ${ }^{13} \mathrm{C}$ NMR (125 MHz, DMSO): $\delta=91.3$ (C-1), 79.8 (C-5), 73.9 (C-3), 68.1 (C-4), 67.5 (C-2), 60.5 (C-6), assignments were confirmed by ${ }^{1} \mathrm{H}^{13}{ }^{13} \mathrm{C}$ HSQC. LRMS $\left(\mathrm{ESI}^{+}\right): \mathrm{m} / \mathrm{z}=266[\mathrm{M}+\mathrm{Na}]^{+}$. LRMS $\left(\mathrm{ESI}^{-}\right): \mathrm{m} / \mathrm{z}$ $=242[\mathrm{M}-\mathrm{H}]^{-}$. HRMS (ESI) calcd for $\mathrm{C}_{6} \mathrm{H}_{13} \mathrm{~N}_{1} \mathrm{O}_{7} \mathrm{SNa}^{+}$: 266.0305, Found: 266.0310.

Methyl 1-S-D-glucopyranuronylsulfonamide (15). To a solution of 11 (173 mg, $0.44 \mathrm{mmol}, 1$ equiv.) in $\mathrm{MeOH}: \mathrm{H}_{2} \mathrm{O}$ (1:4, $7.5 \mathrm{~mL}$ ) was added $\mathrm{NaOH}$ (20 mg, $0.50 \mathrm{mmol}, 1.1$ equiv.). The reaction was stirred for $5 \mathrm{~h}$ at $0{ }^{\circ} \mathrm{C}$ then neutralized with Amberlite resin IR-120 $\mathrm{H}^{+}$, filtered, evaporated and the residue purified by flash chromatography with solid addition (8:2 $\left.\mathrm{CH}_{3} \mathrm{CN}-\mathrm{H}_{2} \mathrm{O}\right)$. Acetonitrile was evaporated from fractions containing the title compound and the remaining aqueous solution filtered (0.42 $\mu \mathrm{m}$ syringe filter) and lyophilized to dryness to furnish the title sulfonamide $\mathbf{1 5}$ as a white solid (66 mg, $0.26 \mathrm{mmol}, 59 \%) . \mathrm{mp}=109-111^{\circ} \mathrm{C}[\alpha]_{\mathrm{D}}^{25}=-18(c=1.1, \mathrm{MeOH}) .{ }^{1} \mathrm{H}$ NMR $\left(400 \mathrm{MHz}, \mathrm{D}_{2} \mathrm{O}\right)$ : $\delta=4.59$ (d, $J=9.6 \mathrm{~Hz}, \mathrm{H}-1), 4.07$ (d, $J=9.2 \mathrm{~Hz}, 1 \mathrm{H}, \mathrm{H}-5), 3.87$ (t, $J=9.2 \mathrm{~Hz}, 1 \mathrm{H}, \mathrm{H}-2), 3.71$ (t, $J=9.2$ $\mathrm{Hz}, 1 \mathrm{H}, \mathrm{H}-3$ ), 3.66 (t, $J=9.2 \mathrm{~Hz}, 1 \mathrm{H}, \mathrm{H}-4$ ) assignments were confirmed by ${ }^{1} \mathrm{H}-{ }^{1} \mathrm{H}$ gCOSY. ${ }^{13} \mathrm{C}$ NMR (125 MHz, DMSO): $\delta=170.2\left(\mathrm{CO}_{2} \mathrm{H}\right), 90.6$ (C-1), 78.7, 76.8, 71.2, 70.6 (C-5, C-2, C-3, C-4). LRMS $\left(\mathrm{ESI}^{+}\right): m / z=280[\mathrm{M}+\mathrm{Na}]^{+}$. HRMS (ESI) calcd for $\mathrm{C}_{6} \mathrm{H}_{11} \mathrm{~N}_{1} \mathrm{O}_{8} \mathrm{SNa}^{+}$: 280.0098, Found: 280.0112.

$\beta$-D-Galactopyranosyl-(1 $\rightarrow$ 4)-1-thio- $\beta$-D-glucopyranosylsulfonamide (16). The title compound was prepared from 12 (462 mg, $0.66 \mathrm{mmol}$ ) as described in general procedure 1 and obtained as an 
orange solid following purification by flash chromatography with solid addition (85:15 $\left.\mathrm{CH}_{3} \mathrm{CN}-\mathrm{H}_{2} \mathrm{O}\right)$.

Acetonitrile was evaporated from fractions containing the title compound and the remaining aqueous solution filtered ( $0.42 \mu \mathrm{m}$ syringe filter) and lyophilized to dryness to furnish the title sulfonamide $\mathbf{1 6}$ as a white solid (144 mg, $0.36 \mathrm{mmol}, 55 \%) . \mathrm{R}_{f}=0.32\left(8: 2 \mathrm{CH}_{3} \mathrm{CN}-\mathrm{H}_{2} \mathrm{O}\right) \cdot \mathrm{mp}=138-139{ }^{\circ} \mathrm{C} \cdot[\alpha]^{25}{ }_{\mathrm{D}}=+13$ $(c=1.0, \mathrm{MeOH}) .{ }^{1} \mathrm{H}$ NMR (500 MHz, DMSO): $\delta=6.71$ (br s, $\left.2 \mathrm{H}, \mathrm{NH}_{2}\right), 5.21(\mathrm{~m}, 1 \mathrm{H}, \mathrm{OH}), 5.06(\mathrm{~d}, J=$ $4.0 \mathrm{~Hz}, 1 \mathrm{H}, \mathrm{OH}$ ), 4.79 (d, $J=1.0 \mathrm{~Hz}, 1 \mathrm{H}, \mathrm{OH}), 4.76$ (m, 1H, OH), 4.63 (t, $J=5.0 \mathrm{~Hz}, 1 \mathrm{H}, \mathrm{OH}$ ), 4.52 (t, $J=6.5 \mathrm{~Hz}, \mathrm{OH}), 4.48$ (d, $J=4.5 \mathrm{~Hz}, 1 \mathrm{H}, \mathrm{OH}), 4.20$ (d, $\left.J=7.5 \mathrm{~Hz}, 1 \mathrm{H}, \mathrm{H}-1^{\prime}\right), 4.13$ (d, $J=9.5 \mathrm{~Hz}, 1 \mathrm{H}$, H-1), 3.80 (m, 1H), 3.62-3.43 (m, 11H) (H-2, H-2', H-3, H-3', H-4, H-4', H-5, H-5', H-6a, H-6a', H-6b, H-6b’), assignments were confirmed by ${ }^{1} \mathrm{H}^{-1} \mathrm{H}$ gCOSY. ${ }^{13} \mathrm{C}$ NMR (125 MHz, DMSO): $\delta=103.7$ (C-1'), 90.0 (C-1), 79.9 (C-4), 78.9 (C-3’), 75.5, 75.4 (C-5, C-5’), 73.2 (C-3), 70.5 (2C) (C-2, C-2’), 68.1 (C4'), 60.5, 60.4 (C-6, C-6'), assignments were confirmed by ${ }^{1} \mathrm{H}^{-13}{ }^{13} \mathrm{C}$ HSQC. LRMS (ESI $\left.{ }^{+}\right): \mathrm{m} / \mathrm{z}=428$ [M $+\mathrm{Na}]^{+}, 833[2 \mathrm{M}+\mathrm{Na}]^{+}$. HRMS (ESI) calcd for $\mathrm{C}_{12} \mathrm{H}_{23} \mathrm{~N}_{1} \mathrm{O}_{12} \mathrm{SNa}^{+}:$428.0833, Found: 428.0836 .

$\alpha$-D-Glucopyranosyl-( $1 \rightarrow 4)$-1-thio- $\beta$-D-glucopyranosylsulfonamide (17). The title compound was prepared from 13 (605 $\mathrm{mg}, 0.86 \mathrm{mmol}$ ) as described in general procedure 1 and obtained as an orange solid following purification by flash chromatography with solid addition $\left(85: 15 \mathrm{CH}_{3} \mathrm{CN}-\mathrm{H}_{2} \mathrm{O}\right)$. Acetonitrile was evaporated from fractions containing the title compound and the remaining aqueous solution filtered ( $0.42 \mu \mathrm{m}$ syringe filter) and lyophilized to dryness to furnish the title sulfonamide $\mathbf{1 7}$ as a white solid (207 mg, $0.51 \mathrm{mmol}, 59 \%) . \mathrm{R}_{f}=0.34\left(8: 2 \mathrm{CH}_{3} \mathrm{CN}-\mathrm{H}_{2} \mathrm{O}\right) \cdot \mathrm{mp}=127-128{ }^{\circ} \mathrm{C} \cdot[\alpha]^{25}=+79$ $(c=0.9, \mathrm{MeOH}) .{ }^{1} \mathrm{H}$ NMR (500 MHz, DMSO): $\delta=6.74$ (br s, $2 \mathrm{H}, \mathrm{NH}_{2}$ ), $5.63(\mathrm{~d}, J=3.0 \mathrm{~Hz}, 1 \mathrm{H}, \mathrm{OH})$, $5.40(\mathrm{~d}, J=6.0 \mathrm{~Hz}, 1 \mathrm{H}, \mathrm{OH}), 5.13(\mathrm{~d}, J=5.5 \mathrm{~Hz}, 1 \mathrm{H}, \mathrm{OH}), 5.04$ (d, $J=4.0 \mathrm{~Hz}, 1 \mathrm{H}, \mathrm{OH}), 4.88$ (d, $J=$ $5.5 \mathrm{~Hz}, 1 \mathrm{H}, \mathrm{OH}$ ), 4.85 (d, $J=4.5 \mathrm{~Hz}, 1 \mathrm{H}, \mathrm{OH}$ ), 4.52-4.46 (m, 2H, OH, H-1'), 4.12 (d, $J=9.0 \mathrm{~Hz}, 1 \mathrm{H}$, H-1), 3.74 (m, 1H), 4.63-3.34 (m, 9H) (H-2, H-2', H-3, H-3', H-4, H-4', H-6a, H-6a', H-6b, H-6b'), 3.23 (m, 1H, H-5), 3.23 (m, 1H, H-5'), assignments were confirmed by ${ }^{1} \mathrm{H}-{ }^{1} \mathrm{H}$ gCOSY.${ }^{13} \mathrm{C}$ NMR (125 MHz, DMSO): $\delta=100.7$ (C-1'), 90.2 (C-1), -1'), 79.9 (C-4), 78.9 (C-3’), 75.5, 75.4 (C-5, C-5'), 73.2 
(C-3), 70.5 (2C) (C-2, C-2'), 68.1 (C-4'), 60.5, 60.4 (C-6, C-6'), assignments were confirmed by ${ }^{1} \mathrm{H}^{13}{ }^{13} \mathrm{C}$ HSQC. LRMS $\left(\mathrm{ESI}^{+}\right): \mathrm{m} / \mathrm{z}=423\left[\mathrm{M}+\mathrm{NH}_{4}\right]^{+}, 428[\mathrm{M}+\mathrm{Na}]^{+}$. LRMS (ESI): $\mathrm{m} / \mathrm{z}=404[\mathrm{M}-\mathrm{H}]^{-}, 410$ $[\mathrm{M}+\mathrm{Cl}]^{-}$. HRMS (ESI) calcd for $\mathrm{C}_{12} \mathrm{H}_{23} \mathrm{~N}_{1} \mathrm{O}_{12} \mathrm{SNa}^{+}$: 428.0833, Found: 428.0823.

\section{Acknowledgement}

This work was financed by the Australian Research Council (Grant number DP0877554); the Eskitis Institute for Cell and Molecular Therapies (Griffith University). We thank A/Prof Scott Taylor for the provision of synthetic details for bis(2,4-dimethoxybenzyl)amine.

\section{Supporting Information Available}

Detailed experimental procedures, characterization data, and copies of ${ }^{1} \mathrm{H}$ and ${ }^{13} \mathrm{C}$-spectra of new compounds $4, \mathbf{5}, \mathbf{1 0 - 1 7}$. This material is available free of charge via the Internet at http://pubs.acs.org

\section{References}

1. Njardarson, J.T.; Cornell University; Outreach Web Page. http://www.chem.cornell.edu/jn96/outreach.html (accessed November 2008).

2. (a) Anand, N. Burger's Medicinal Chemistry and Drug Discovery, 5th ed.; Wollf, M.E., Ed.; John Wiley \& Sons: New York, 1996; Vol. 2, pp 527. (b) Supuran, C.T. Nat. Rev. Drug Disc. 2008, 7, 168.

3. Knapp, S.; Darout, E.; Amorelli, B. J. Org. Chem. 2006, 71, 1380.

4. (a) Owen, D.J.; von Itzstein, M. Carbohydr. Res. 2000, 328, 287. (b) Owen, D.J.; Davis, C.B.; Hartnell, R.D.; Madge, P.D.; Thomson, R.J.; Chong, A.K.J.; Coppel, R.L. von Itzstein, M. Bioorg. Med. Chem. Lett. 2007, 17, 2274. 
5. Zemplen, G. Ber. Deutsch. Chem. Ges. 1926, 59, 1254.

6. (a) Hill, B.; Liu, Y.; Taylor, S.D. Org. Lett. 2004, 6, 4285. (b) Hill, B.; Ahmed, V.; Bates, D.; Taylor, S.D. J. Org. Chem. 2006, 71, 8190. (c) Navidpour, L.; Lu, W.; Taylor, S.D. Org. Lett. 2006, 8, 5617. (d) Chen, L.; Gao, G.; Bonnac, L.; Wilson, D.J.; Bennett, E.M.; Jayaram, H.N.; Pankiewicz, K.W. Bioorg. Med. Chem. Lett. 2007, 17, 3152.

7. Kocienski, P.J. Protecting Groups, 2nd ed.; Thieme: Stuggart, 2000.

8. Agnihotri, G.; Misra, A.K. Carbohydr. Res. 2006, 341, 275.

9. Ibatullin, F.M.; Shabalin, K.A.; Jänis; J.V.; Shavvac, A.G. Tetrahedron Lett. 2003, 44, 7961.

\section{TOC Graphic}

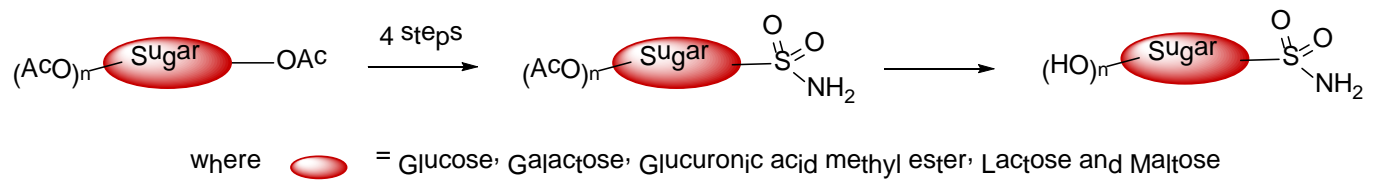

